\title{
Sistematización de asesoría telefónica en un centro de información toxicológica auxiliada por fichero
}

\section{Systematization of telephone counseling in a toxicology information center assisted by file}

\author{
Lilia E. Pozas-Ocampo ${ }^{*}$, Patricia Escalante-Galindo ${ }^{1}$, Jesús del C. Madrigal ${ }^{1}$, Miguel A. Canul-Caamal', \\ Mario A. Moreno-Eutimio ${ }^{2}$ y Daniel A. Juárez-Ascencio ${ }^{2}$ \\ ${ }^{1}$ Centro de Información y Asistencia Toxicológica; ${ }^{2}$ Coordinación de Investigación Clínica. Hospital Juárez de México, Ciudad de México, México
}

\begin{abstract}
Resumen
Antecedentes: La telemedicina es reconocida por la Organización Mundial de la Salud (OMS) como la práctica de cuidado de la salud que usa comunicaciones interactivas. Derivan de aquí los conceptos de teleconsulta y teleasistencia. Estos recursos han facilitado la accesibilidad, eficiencia y el ahorro de gastos en la atención de los pacientes intoxicados. Objetivo: Sistematizar la asesoría telefónica en un centro de información toxicológica por medio del uso de ficheros de información. Material y métodos: Tipo de estudio: transversal, retrospectivo. Lugar: Centro de Información y Asistencia Toxicológica del Hospital Juárez de México, Ciudad de México. Sujetos: Llamadas registradas en bitácora del Centro de Información y Asistencia Toxicológica durante un periodo de 1 año. Procedimientos: 1. Revisión de la bitácora de llamadas telefónicas realizadas durante el periodo de estudio. 2. Construir una base de datos en Excel 3. Análisis estadístico de información. 3. Diseñar al menos 10 fichas de manejo de las intoxicaciones más comunes. 4. Validación de las fichas por dos expertos en toxicología. Resultados: Se registraron 280 asesorías en 3 años. La intoxicación que más se asesoró fue por animales de ponzoña, específicamente: Ioxoscelismo, arañas, ofidismo y alacrán. Se realizaron fichas con las intoxicaciones más comunes y se diseñó el software con la información. Conclusiones: Las asesorías telefónicas son una herramienta importante en la adecuada atención del paciente intoxicado, por lo que se les debe dar más importancia y difusión.
\end{abstract}

Palabras clave: Telemedicina. Teleconsulta. Toxicología. Asesoría toxicológica.

\section{Abstract}

Background: Telemedicine is recognized by WHO as the practice of health care using interactive communications. From here derive the concepts: Teleconsultation and Telecare. These resources have facilitated accessibility, efficiency and cost savings in the care of intoxicated patients. Objective: To systematize telephone counseling in a toxicological information center through the use of information files. Material and methods: Type of study: Cross-sectional, descriptive, observational, retrospective. Place: Information and Toxicological Assistance Center of the Hospital Juárez de México. Subjects: Calls registered in a log for 3 years. Procedures: (1) Review of logbook. (2) Build a database in Excel with each variable (3) Statistical analysis. (4) Design management sheets of the most common poisonings. (5) Validation of the cards by 2 toxicologists. Results: 280 consultancies were registered in the course of 3 years. The poisoning that was most advised was by animals of poison, specifically: Loxoscelismo, spiders, office and alacran. Ten information sheets were made and validated for their use. Conclusions: Telephone

Correspondencia:

*Lilia E. Pozas-Ocampo

E-mail: liliaelizabeth@gmail.com

DOI: 10.24875/RHJM.M20000005
Disponible en internet: 10-03-2020 Rev Hosp Jua Mex. 2020;87(1):21-27

www.revistahospitaljuarez.com licencia CC BY-NC-ND (http://creativecommons.org/licenses/by-nc-nd/4.0/). 
counseling is currently an important tool in the adequate care of the intoxicated patient, decreases costs in care and avoids errors in treatment. The toxicological information file is a tool that seeks to ensure that information is provided more efficiently.

Key words: Telemedicine. Teleconsultation. Toxicology. Toxicological advice.

\section{Introducción}

A lo largo de la historia, los medios de comunicación se han adaptado a las diferentes necesidades del ser humano, así mismo, han evolucionado junto con él con ayuda de la tecnología, que se ha vuelto cada vez más accesible e indispensable. Es así como surge la telemedicina, que es considerada por la Organización Mundial de la Salud como la práctica de cuidado de la salud que usa comunicaciones interactivas de audio, visuales y datos médicos ${ }^{1}$. La Asociación Americana de Telemedicina la define como utilización de datos clínicos intercambiados de un sitio a otro a través de comunicación electrónica para mejorar el estado de salud clínica de un paciente ${ }^{2}$.

La telemedicina incluye una variedad muy amplia de información, como un video interactivo, fotos, monitoreo de video, correos electrónicos, llamadas telefónicas, etc. Beneficia a muchos niveles: agiliza las acciones de la atención médica, facilita la convivencia entre médicos de primer contacto con especialistas, lo que crea un espíritu de trabajo en equipo al tener una asesoría directa, evita referencias innecesarias a un segundo nivel, lo que reduce gastos, optimiza la atención de los pacientes, evita cometer errores y disminuye las complicaciones potenciales. Es una herramienta accesible, ya que cada vez los medios de comunicación se van universalizando, poco a poco se va facilitando la comunicación en casi todos niveles ${ }^{3-5}$.

No se tiene bien claro en qué momento surgió la telemedicina, casi podemos estar seguros que surge al mismo tiempo en el que los medios de comunicación electrónicos empiezan a existir, ya que la salud para el ser humano siempre ha sido una prioridad. Actualmente se cuenta con registros de varias situaciones en donde la telemedicina se ha aplicado para facilitar la atención a la salud, desde asesoría de personal médico para astronautas de la primera tripulación a la luna en 1969 en la NASA (National Aeronautics and Space Administration) hasta la asesoría de una operadora que puede detectar datos clínicos de paro cardiorrespiratorio y guiar una reanimación cardiopulmonar básica en el servicio 911, recomendada por las guías Advanced Cardiovascular Life Support (ACLS) de la American Heart Association (AHA) ${ }^{6}$.
Hablando de toxicología, en 1966, el Dr. Eduardo Picazo Michel crea el primer Centro de Información Toxicológica (CIT) en el Hospital de Pediatría del Centro Médico Nacional del Instituto Mexicano del Seguro Social, por lo cual se le considera fundador de la toxicología en México. Puntualiza la importancia de los CIT como "un organismo que ofrezca información al médico o a otra persona acerca de los componentes tóxicos de cualquier sustancia, que proporcione indicaciones terapéuticas, que, inclusive, posea facilidades hospitalarias que le permitan llevar a cabo tratamiento especializado de los casos de intoxicaciones y que a la vez pueda, con base en su propia experiencia, dictar las medidas preventivas más útiles»?.

En el año 2000 se crea la agrupación RETOMEX con la finalidad de agrupar CIT. Crean un directorio, conformado por 24 instituciones que brindan asesorías toxicológicas en todo el país, de las cuales, 18 son CIT. En un reporte epidemiológico de 2005, en nuestro país, las intoxicaciones y envenenamientos provocaron 13,600 egresos hospitalarios. Si dividimos esta cantidad de intoxicados entre los CIT, a cada uno le tocaría atender a 755.5 intoxicados ${ }^{7,8}$. Lo anterior, definitivamente no es factible, ya que los pacientes no se presentan de manera uniforme geográficamente. Muchos pacientes son atendidos en áreas de urgencias de centros hospitalarios, que en su mayoría no cuentan con servicio de toxicología.

El Centro de Información y Asistencia Toxicológica (CIAT) del Hospital Juárez de México, fundado en el año 2007, además de ser centro de referencia de pacientes a nivel nacional, también es un centro de asesoría para la atención del paciente intoxicado. Las asesorías se llevan a cabo las 24 horas del día, los 365 días del año y son realizadas principalmente por médicos becarios que, a su vez, son coordinados por médicos toxicólogos adscritos y por la propia Dra. Patricia Escalante Galindo, Jefa del CIAT. A lo largo de más de 10 años de existencia del CIAT se ha acumulado una importante casuística de asesorías, mediante el registro de la información solicitada.

\section{Material y métodos}

Se realizó un estudio transversal, descriptivo y retrospectivo. Se revisó la bitácora de asesorías 


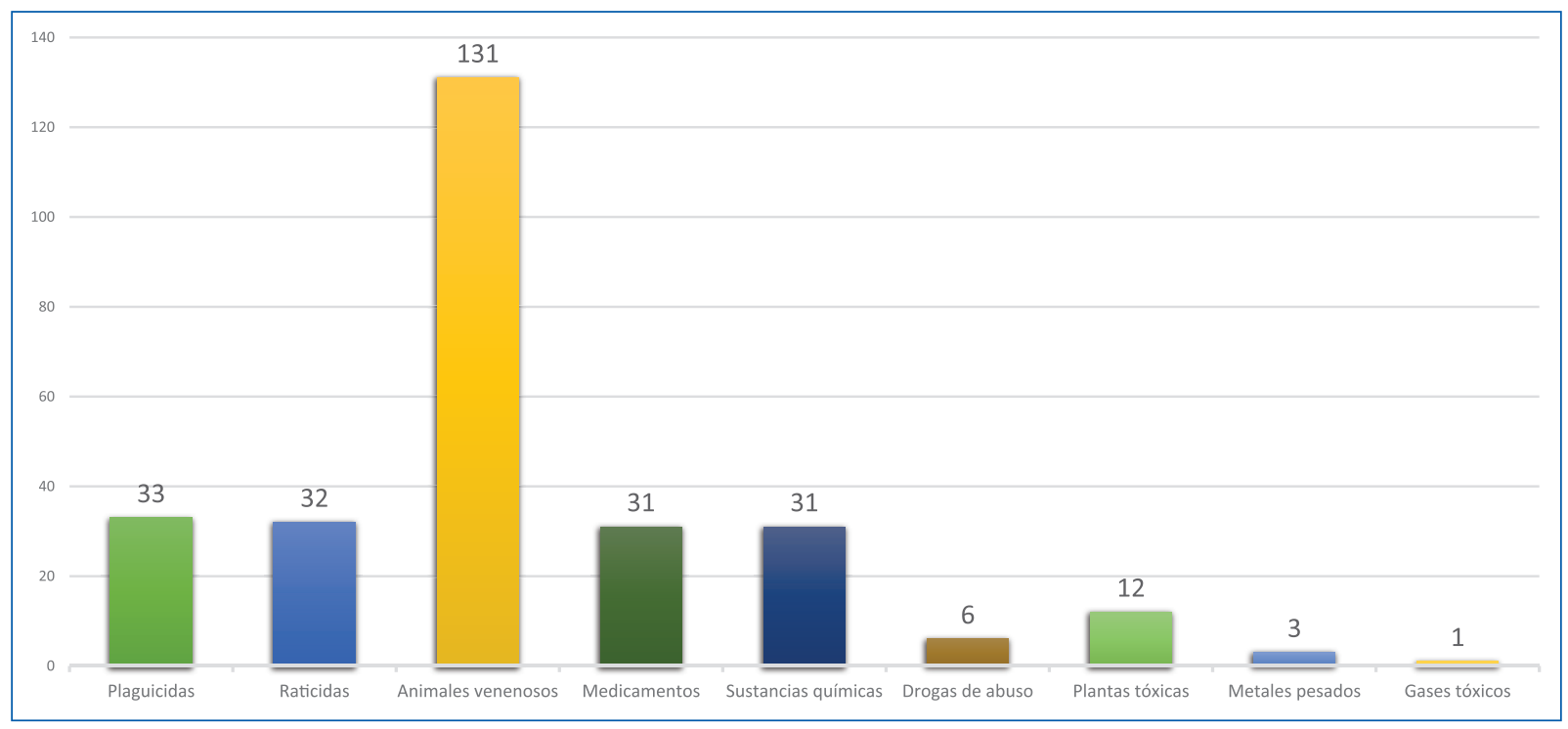

Figura 1. Número de asesorías otorgadas por vía telefónica clasificadas por tipo de xenobiótico.

telefónicas realizadas del 2016 al 2018 en el CIAT del Hospital Juárez de México. Se concentró la información en una base de datos con las siguientes variables: tipo de xenobiótico, solicitante de asesoría, edad del intoxicado, género del intoxicado, fecha de la asesoría. Se utilizó la herramienta digital de estadística SPSS versión 2.22. Para variables cualitativas se calcularon frecuencias relativas y absolutas; para las cuantitativas, se calcularon medidas de tendencia central y dispersión apropiadas, dependiendo la distribución de las variables con respecto a la normalidad. Una vez establecido el perfil de las intoxicaciones más asesoradas, se diseñaron fichas de información con algoritmos de abordaje, las cuales fueron validadas por dos expertos toxicólogos.

\section{Resultados}

El total de asesorías registradas durante 3 años fueron 280. Las asesorías se solicitaron desde diferentes puntos geográficos, tanto dentro de nuestra misma ciudad $(33 \%)$ como de otros países $(0.7 \%)$, específicamente de Barcelona y de Perú. Los estados fueron muy variados, sobresaliendo Morelos, Puebla e Hidalgo.

Los solicitantes de las asesorías se dividieron en médicos y familiares del intoxicado. Los que más solicitaron asesorías fueron los médicos: 219 , y los familiares solicitaron 61 .

En cuanto a los intoxicados que generaron las asesorías, la mayoría fueron del género masculino: 164 .
Las edades de los intoxicados variaron entre un mínimo de 15 días hasta 79 años, con una media de 26.40 años y una desviación estándar de 20-21.

Las asesorías solicitadas fueron de diferentes xenobióticos, los cuales se clasificaron en nueve categorías diferentes. La categoría que más se registró fue la de animales venenosos, de estos, el loxoscelismo fue el envenenamiento más asesorado, seguido por ofidismo y alacranismo (Fig. 1).

Con base en esta información diseñamos diez fichas de información para auxiliar las asesorías, de tal manera que fueran comprensibles para médicos o personal de salud (Figs. 2 a 6$)^{9-36}$

\section{Discusión}

Las asesorías se realizaron todos los meses del año. Son solicitadas desde diferentes puntos geográficos, la mayoría del Estado de México, y desde diferentes hospitales o instituciones de salud. Solo se consideraron los registros de asesorías por llamado telefónico, aunque actualmente las asesorías también se realizan por las redes sociales.

La mayor parte de los intoxicados se encontraba en urgencias al momento de solicitar la asesoría. Esto lo consideramos importante, ya que de esta manera se asegura otorgar información sobre la adecuada atención desde el primer contacto con el intoxicado, sobre todo cuando sabemos que en algunas intoxicaciones 


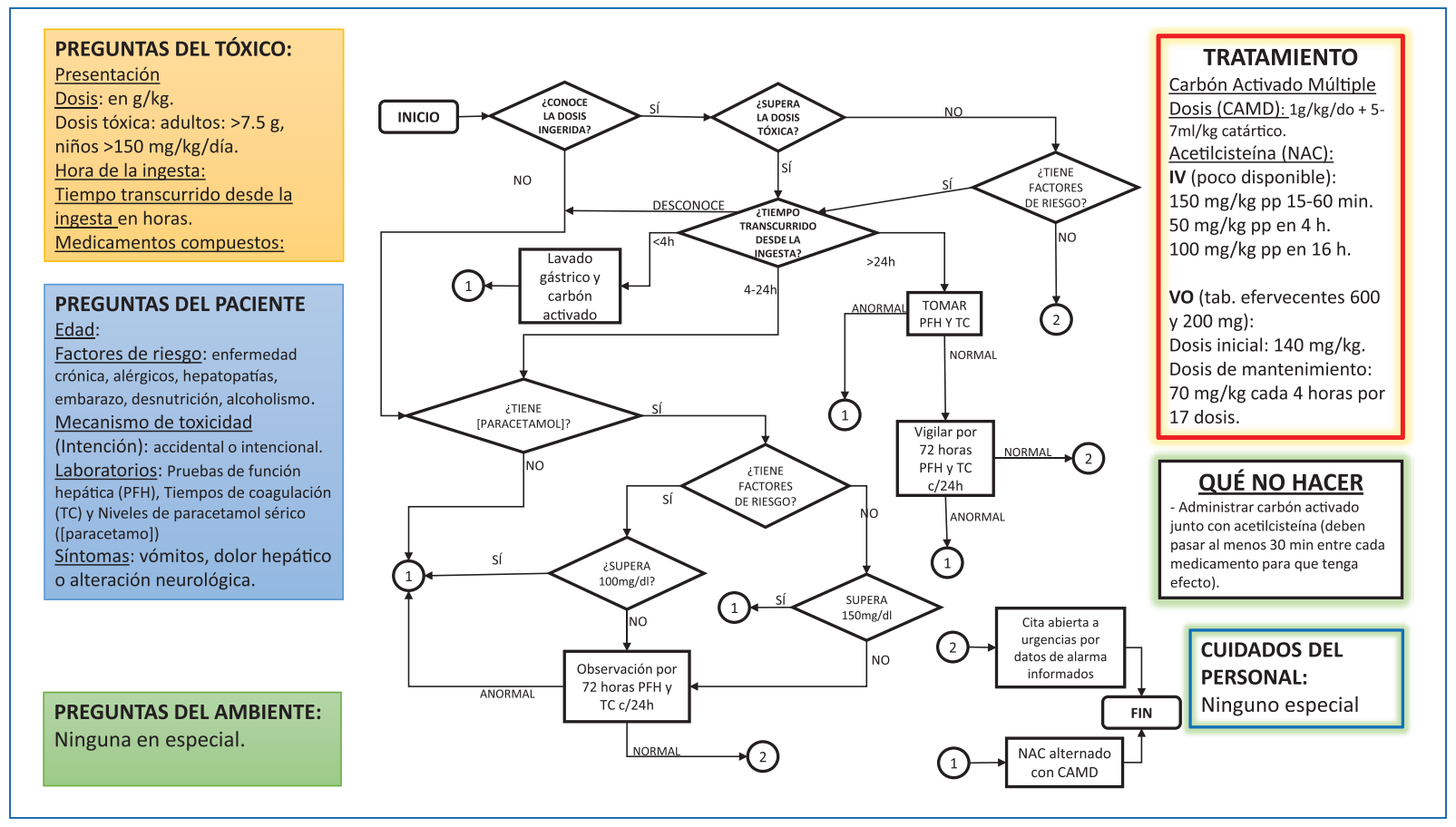

Figura 2. Ficha de información para la asesoría de intoxicación por paracetamol.

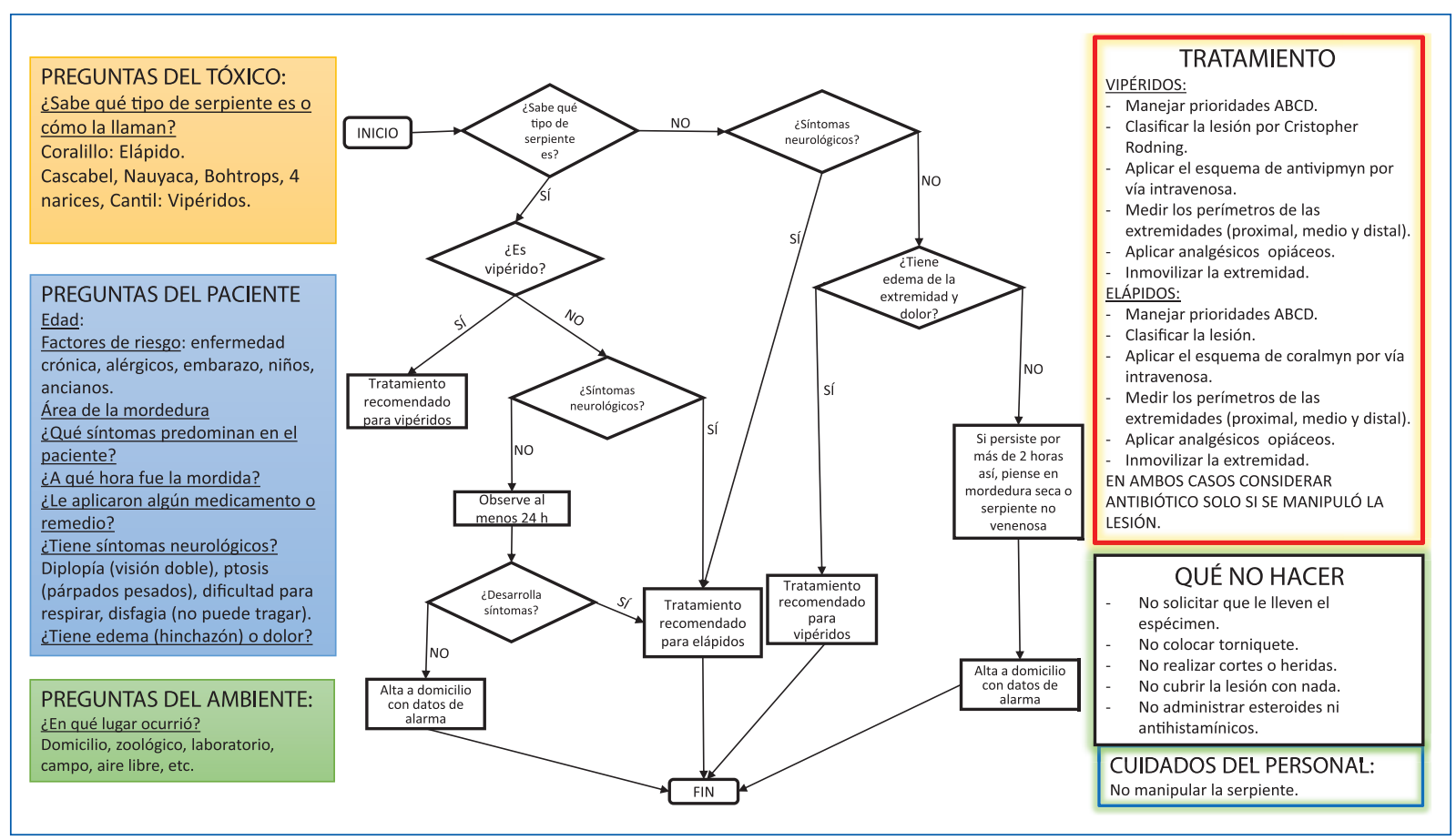

Figura 3. Ficha de información toxicológica de ofidismo.

el pronóstico puede cambiar si no se realizan algunas acciones específicas. Tomando en cuenta esto, el fichero se diseñó de tal manera que se oriente al médico sobre qué medidas se deben tomar como parte de la adecuada atención y, lo más importante, qué acciones no realizar para que se asegure un buen pronóstico del paciente, tomando en consideración también la seguridad del personal sanitario y no 


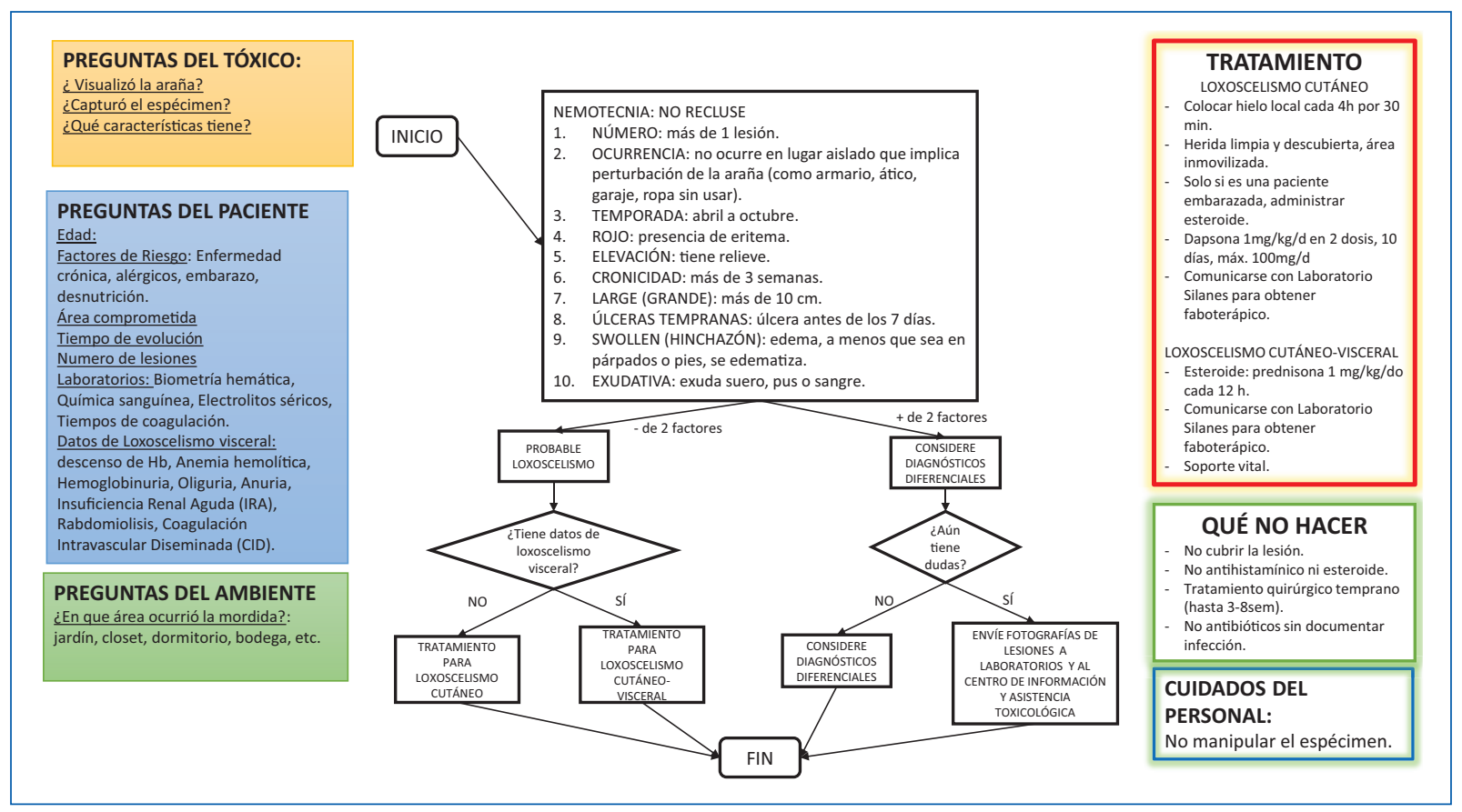

Figura 4. Ficha de información toxicológica de loxoscelismo.

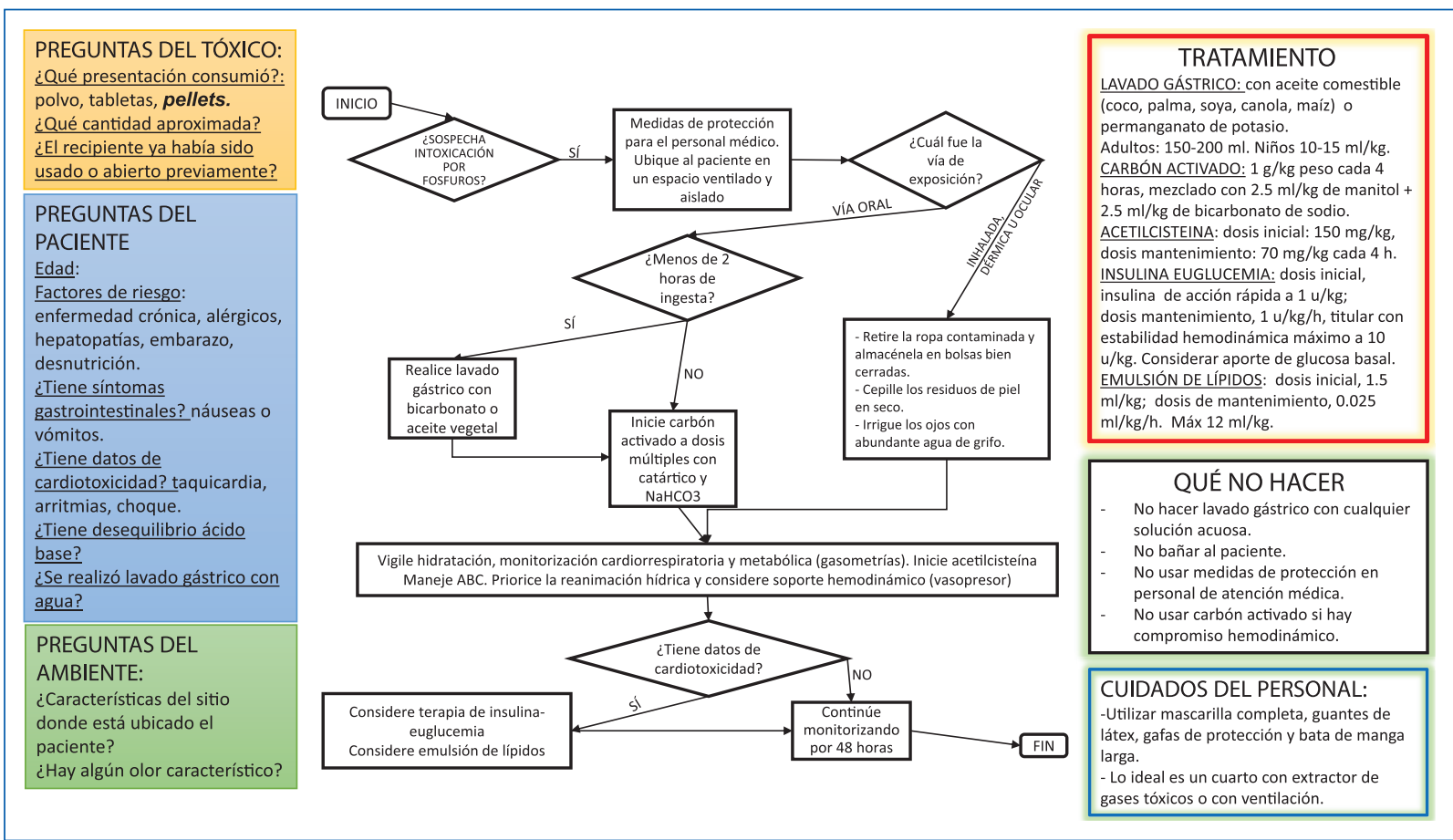

Figura 5. Ficha de información de intoxicación por rodenticidas tipo fosfuros.

dejando a un lado la posibilidad de trasladar al paciente una vez que mejoren sus condiciones generales.

No todos los solicitantes de las asesorías son médicos, un $22 \%$ fueron solicitadas por familiares, por lo que algunas consideraciones del fichero se realizaron para personas no relacionadas con cuidados de la salud, haciendo énfasis en acudir en breve a una valoración médica, ya que, como bien sabemos, la valoración de 


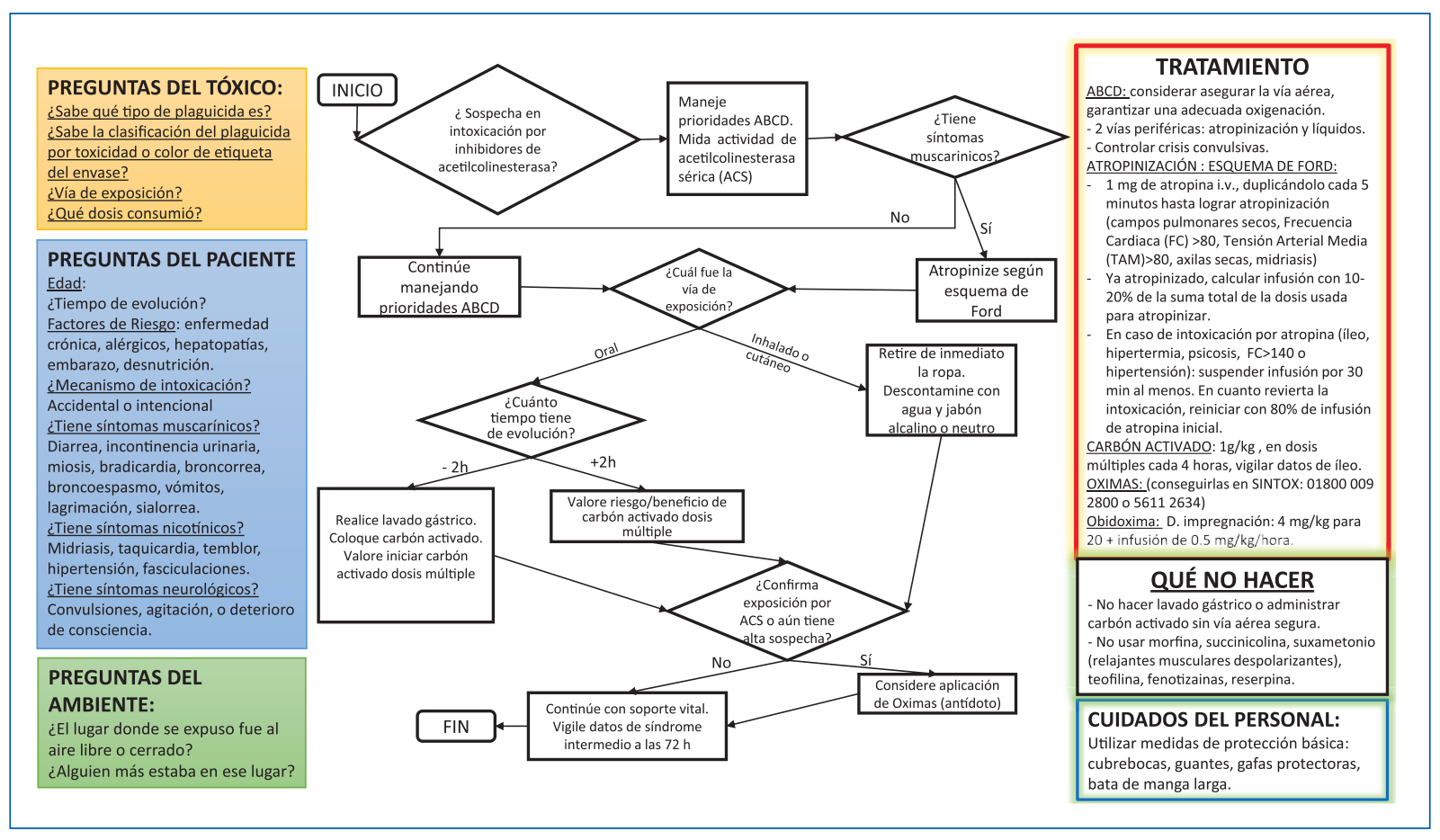

Figura 6. Ficha de información de intoxicación por organofosforados y carbamatos.

cualquier padecimiento no se sustituye con una sola asesoría telefónica. Específicamente se puntualiza más en las acciones que no deben realizarse para modificar la evolución o empeorarla.

Destacamos que dentro de este conteo se incluye a un veterinario que solicitó una asesoría para un perro macho de 3 años de edad, el cual se intoxicó por un raticida de tipo fosfuro de zinc. Al no tener una categoría especial y ser el único caso de este tipo, se incluyó dentro de toda la estadística. En este caso, la asesoría otorgada se basó en los cuidados básicos de soporte para el animal y protección para el veterinario y los cuidadores. Algunas acciones de las fichas también pueden acoplarse a cuidados veterinarios.

Los xenobióticos fueron clasificados en nueve categorías, los más comunes fueron los animales venenosos, seguidos por plaguicidas, raticidas, medicamentos, sustancias químicas, etc. En otros centros toxicológicos, como el Centro de Información Toxicológica de la Universidad Católica de Chile (CITUC) o el Centro Toxicológico de Bogotá, se reporta como xenobiótico más común la categoría de medicamentos. Esto se puede explicar por la biodiversidad que existe en nuestro país, de tal manera que, incluso en áreas urbanas, encontramos animales venenosos que generan lesiones.

Toda esta información se utilizó para diseñar las fichas de consulta según lo más demandado en las asesorías. Es importante aclarar que este trabajo es solo el inicio de lo que se espera sea una base de datos bastante amplia para que cualquier intoxicación pueda ser asesorada por cualquier personal, sin importar que tenga o no una preparación previa en toxicología.

\section{Conclusión}

Los medios de comunicación van evolucionando a lo largo de los tiempos, logrando mejorar y facilitar el intercambio de información en diferentes situaciones de la vida diaria. No es la excepción el caso de la salud. La toxicología es una especialidad a la cual aún no se le ha dado la importancia suficiente en los diferentes sectores de salud. La administración hospitalaria en nuestro país no toma la toxicología como una especialidad básica en la oferta de especialidades de hospitales, de aquí la importancia de contar con centros de asesoría toxicológica con la organización necesaria para suplir esta falta de toxicólogos. Si bien la educación médica continua es una gran fortaleza, las dudas pueden surgir en todo momento durante la atención inicial del intoxicado.

El fichero toxicológico pretende ser una herramienta para que cualquier personal médico realice la asesoría de manera puntual y eficiente, y que garantice un mejor pronóstico de los pacientes intoxicados y que minimice los riesgos tanto de personal de salud como de los familiares o testigos. Fue avalado por dos toxicólogos 
del CIAT del Hospital Juárez de México (Dr. Jesús Madrigal Anaya y Dr. Miguel Antonio Canul Caamal), los cuales, con su experiencia, enriquecieron esta base de datos que se espera sea el inicio de una base de información mucho más amplia para asegurar que cualquier intoxicación que requiera de asesoría sea facilitada.

Esta investigación es la primera que se realiza de este tipo en el CIAT del Hospital Juárez de México, y se convierte en un referente del comportamiento epidemiológico de las intoxicaciones que demandan asesoría toxicológica.

\section{Conflicto de intereses}

Los autores declaran no tener conflicto de intereses alguno.

\section{Financiamiento}

La presente investigación no ha recibido ninguna beca específica de agencias de los sectores público, comercial, o sin ánimo de lucro.

\section{Responsabilidades éticas}

Protección de personas y animales. Los autores declaran que los procedimientos seguidos se conformaron a las normas éticas del comité de experimentación humana responsable y de acuerdo con la Asociación Médica Mundial y la Declaración de Helsinki.

Confidencialidad de los datos. Los autores declaran que en este artículo no aparecen datos de pacientes.

Derecho a la privacidad y consentimiento informado. Los autores declaran que en este artículo no aparecen datos de pacientes.

\section{Bibliografía}

1. Braun R, Vecchietti J, Thomas L, Prins C, French L, Gewirtzman A, et al. Telemedical Wound Care Using a New Generation of Mobile Telephones. Arch Dermatol. 2005;141(2):254-8.

2. Gaynor M. Evaluation of Patient to Provider Oriented Telemedicine in Hospitals and Physician Practices. Muskie School Capstones. (Internet) 2015 (citado el 29 marzo 2018). Disponible en: http://digitalcommons. usm.maine.edu/muskie_capstones/103.

3. Sanchez JA. Aspectos Éticos y Médico-Legales en la Telemedicina: La consulta Médica Telefónica (Tesis de Doctorado). Madrid: Universidad Complutense de Madrid; 2011. p. 120.

4. Bernal G, de la Torre J, Robles C, Campos A, Otero C. Análisis costo-beneficio. Telemedicina Anáhuac. Cirugía y Cirujanos [Internet]. 2007;75(3):227-35. Recuperado de: http://www.redalyc.org/articulo. oa? id=66275314

5. Olarte M, Espinoza A, Suarez D. Asesorías telefónicas en un servicio de toxicología 2006-2009. Rev Salud Publica. 2011;13(4):644-53.

6. Aspectos Destacados de la Actualización de las Guías de la AHA para RCP y ACE de 2015. (Internet). Dallas, Texas: American Hearth Asociación. 2015; (Citado el 30 de marzo de 2018). Disponible en: https://eccguidelines.heart.org/wp-content/uploads/2015/10/2015-AHA-Guidelines-Highlights-Spanish.pdf
7. Víquez JE. IMSS. (Internet). Centro de Información y Asistencia Toxicológica. Antecedentes. (Citado el 1 de abril de 2018). Disponible en: http:// edumed.imss.gob.mx/pediatria/toxico/pagtoxico.htm

8. Hoffman R, Howland M, Lewin N, Nelson L, Goldfrank L, Flomenbaum N. Goldfrank's toxicologic emergencies. 10 $0^{\text {th }}$ ed. New York: McGraw Hill; 2015.

9. Chiew A, Isbister G, Kirby K, Page C, Chan B, Buckley N. Massive paracetamol overdose: an observational study of the effect of activated charcoal and increased acetylcysteine dose (ATOM-2). Clin Toxicol (Phila). 2017;55(10):1055-65.

10. Schwarz E, Cohn B. Is Intravenous Acetylcysteine More Effective Than Oral Administration for the Prevention of Hepatotoxicity in Acetaminophen Overdose? Ann Emerg Med. 2014;63(1):79-80.

11. Green JL, Heard KJ, Reynolds KM, Albert D. Oral and Intravenous Acetylcysteine for Treatment of Acetaminophen Toxicity: A Systematic Review and Meta-analysis. West J Emerg Med 2012;XIV(3):218-23.

12. Pettie J, Dow M, Sandilands E, Thanacoody H, Bateman D. An integrated care pathway improves the management of paracetamol poisoning. Emerg Med J. 2011;29(6):482-6.

13. A multicenter comparison of the safety of oral versus intravenous acetylcysteine for treatment of acetaminophen overdose. Clin Toxicol (Phila). 2010;48(5):424-30.

14. Agrawal P, Brown C. An Evidence-Based Approach to acetaminophen (Paracetamol, APAP) Overdose. Emergency Medicine Practise. 2010; 12(9):1-25.

15. Secretaría de Salud. Diagnóstico y tratamiento de la Intoxicación Aguda por Acetaminofén. México: Secretaría de Salud; 2010.

16. Lavonas E, Ruha A, Banner W, Bebarta V, Bernstein J, Bush S, et al. Unified treatment algorithm for the management of crotaline snakebite in the United States: results of an evidence-informed consensus workshop. BMC Emergency Medicine. 2011;11(1):49.

17. Zúñiga IR, Lozano JC. Aspectos clínicos y epidemiológicos de la mordedura de serpientes en México. Evid Med Invest Salud. 2013;6(4):125-36.

18. Agency for Clinical Innovation. Snakebite and Spiderbite Clinical Management Guidelines 2013. $3^{\text {th }}$ ed. Public Health System, Divisions of General Practice, Government Medical Officers, Ministry of Health, Private Hospitals and Day Procedure Centres, Tertiary Education Institutes; 2014.

19. González A, Chico P, Domínguez W, Iracheta M, López M, Cuellar A. Epidemiología de las mordeduras por serpiente. Su simbolismo. Acta Pediatr Mex. 2009;30(3):182-91.

20. Secretaría de Salud. Diagnóstico y Tratamiento de las Mordeduras de Serpientes Venenosas. México: Secretaría de Salud; 2010.

21. Amate Blanco JM, Conde Espejo P. (Coord.). Intoxicaciones por mordeduras de ofidios venenosos (I Panel de expertos en España). Madrid: Agencia de Evaluación de Tecnologías Sanitarias (AETS);2012/68.

22. Swanson DL, Vetter R. Bites of Brown Recluse Spiders and Suspected Necrotic Arachnidism. N Engl J Med. 2005;352(7):700-7.

23. Elston DM, Miller MSD, Young RJ, Eggers J, McGlasson MS, Schmidt WH, et al. Comparison of Colchicine, Dapsone, Triamcinolone, and Diphenhydramine Therapy for the Treatment of Brown Recluse Spider Envenomation. Arch Dermatol. 2005;141:595-57.

24. Secretaría de Salud. Diagnóstico y Tratamiento de las Mordeduras Mordeduras de Arañas Venenosas. México: Secretaría de Salud; 2011.

25. Faboterapia. Guía de tratamiento en intoxicaciones por animales ponzoñosos. Instituto Bioclon. Silanes. Redtox.

26. De Roodt A, Salomón O, Lloveras S, Orduña T. Envenenamiento por arañas del genero Loxosceles. Medicina (Buenos Aires). 2002;62:83-94.

27. Stoecker W, Vetter R, Dyer J. NOT RECLUSE-A Mnemonic Device to Avoid False Diagnoses of Brown Recluse Spider Bites. JAMA Dermatology. 2017;153(5):377.

28. Chaudhry $D$, Raí $A$. N-acetyl cysteine in aluminum phosphide poisoning: Myth or hope. Indian J Critic Care Med. 2014;18(10):646.

29. Moghadamnia A. An update on toxicology of aluminum phosphide. Daru. 2012;20(1):25.

30. Anand R, Binukumar B, Gill K. Aluminum phosphide poisoning: an unsolved riddle. J Applied Toxicol. 2011;31(6):499-505.

31. Mehrpour O, Jafarzadeh M, Abdollahi M. A Systematic Review of Aluminium Phosphide Poisoning. Archives of Industrial Hygiene and Toxicology. 2012;63(1):61-73.

32. Secretaría de Salud. Prevención primaria, diagnóstico precoz y tratamiento oportuno de la intoxicación aguda por agroquímicos en el primer nivel de atención. México: Secretaría de Salud; 2008.

33. Virú Loza M. Manejo actual de las intoxicaciones agudas por inhibidores de la colinesterasa: conceptos erróneos y necesidad de guías peruanas actualizadas. An de Fac Med. 2016;76(4):431.

34. Eddleston M, Dawson A, Karalliedde L, Dissanayake W, Hittarage A Azher S, et al. Early management after self-poisoning with an organophosphorus or carbamate pesticide - a treatment protocol for junior doctors. Critical Care. 2004;8(6):R391.

35. Eddleston M, Buckley N, Checketts H, Senarathna L, Mohamed F, Sheriff $M$, et al. Speed of Initial Atropinisation in Significant Organophosphorus Pesticide Poisoning-A Systematic Comparison of Recommended Regimens. J Toxicology: Clin Toxicol. 2004;42(6):865-75. 